Cinémas

Revue d'études cinématographiques

Journal of Film Studies

\title{
La réception du Dernier Métro et de La Femme d'à côté en U.R.S.S. : la stratégie truffaldienne de l'auteurisme face au consensus idéologique
}

\section{Laurence Alfonsi}

Volume 7, numéro 1-2, automne 1996

URI : https://id.erudit.org/iderudit/1000940ar

DOI : https://doi.org/10.7202/1000940ar

Aller au sommaire du numéro

\section{Éditeur(s)}

Cinémas

ISSN

1181-6945 (imprimé)

1705-6500 (numérique)

Découvrir la revue

Citer cet article

Alfonsi, L. (1996). La réception du Dernier Métro et de La Femme d'à côté en U.R.S.S. : la stratégie truffaldienne de l'auteurisme face au consensus idéologique. Cinémas, 7(1-2), 205-224. https://doi.org/10.7202/1000940ar

\section{Résumé de l'article}

L'article se propose de montrer comment, pat leur caractère subversif, les deux films de François Truffaut, Le Dernier Métro et La Femme d'à côté, peuvent bousculer les conventions et le consensus idéologiques. La stratégie artistique de Truffaut d'" appel aux particularismes " prend tout son poids dans des moments de crises comme la pré-perestroïka, où elle devient

l'anti-idéologisation. En quoi les deux films de Truffaut peuvent apparaître comme une menace à l'idéologie et comme une arme pour la libéralisation des années quatre-vingt? Le rôle du cinéma truffaldien est d'autant plus significatif que Le Dernier Métro et La Femme d'à côté constituent deux films thématiquement très différents. Ainsi, l'auteure montre comment l'identification souterraine du public est amenée à s'effectuer sur la base de la dualité, de l'officieux, de l'illicite. Elle correspond ainsi parfaitement à la stratégie truffaldienne qui développe l'ambivalence, l'apparence « civile » en même temps que la face personnelle et cachée. 


\title{
La réception du Dernier Métro et de La Femme d'à côté en U.R.S.S. : la stratégie truffaldienne de l'auteurisme face au consensus idéologique
}

\section{Laurence Alfonsi}

\begin{abstract}
RÉSUMÉ
L'article se propose de montrer comment, par leur caractère subversif, les deux films de François Truffaut, Le Dernier Métro et La Femme d'à côté, peuvent bousculer les conventions et le consensus idéologiques. La stratégie artistique de Truffaut d'"appel aux particularismes" prend tout son poids dans des moments de crises comme la pré-perestroïka, où elle devient l'antiidéologisation. En quoi les deux films de Truffaut peuvent apparaître comme une menace à l'idéologie et comme une arme pour la libéralisation des années quatre-vingt? Le rôle du cinéma truffaldien est d'autant plus significatif que Le Dernier Métro et La Femme d'à côté constituent deux films thématiquement très différents. Ainsi, l'auteure montre comment l'identification souterraine du public est amenée à s'effectuer sur la base de la dualité, de l'officieux, de l'illicite. Elle correspond ainsi parfaitement à la stratégie truffaldienne qui développe l'ambivalence, l'apparence "civile" en même temps que la face personnelle et cachée.
\end{abstract}

\section{ABSTRACT}

This article suggests how, by their subversive character, two films by François Truffaut, Le Dernier Métro and La Femme d'à côté, upset conventions and ideological 
consensus. Truffaut's artistic strategy of the "appeal to particularisms" attains its full strength at times of crisis like the pre-Perestroika period, when it becomes antiideologization. In what way can these two films by Truffaut be seen as a threat to ideology and as a weapon of liberalization in the 1980 s? The subversive role of Truffaut's cinema is all the more significant in that Le Dernier Métro and La Femme d'à côté are thematically very different. Thus the author shows how the subterranean identification of the public is brought about on the basis of duality, the unofficial, the illicit. This corresponds perfectly to the Truffaut strategy of fostering ambivalence, with the appearance of " civility" obscuring the hidden, personal face.

En Europe centrale et orientale, les films de François Truffaut s'imposèrent par une stratégie de l'individualisme, de l'originalité, qui bouscule les conventions idéologiques. Afin d'étudier les conséquences de la stratégie truffaldienne sur le débat critique puis sur la détermination d'un désir d'identification, nous nous appuierons ici sur la réception critique soviétique du Dernier Métro (1980) et de La Femme d'à côté (1981). Nous montrerons comment les films de Truffaut parviennent à s'appuyer sur l'obstacle politique, ce qui, loin de les desservir, les insère dans une dialectique qui accroît leur impact subreptice. La stratégie artistique de Truffaut "d'appel aux particularismes" prenait tout son poids dans des moments de crises où elle apparaissait comme l'anti-idéologisation. Aussi, nous nous demanderons en quoi le film peut apparaître comme une menace à l'idéologie et comme une arme pour la libération des années quatre-vingt.

De 1968 à 1984, une période de stagnation se met en place avec un sévère contrôle idéologique. Les interprétations soviétiques des films de François Truffaut sont déterminées par l'opposition entre l'idéologie de masse et la culture individuelle, et l'identification comme la dénonciation passe par cette opposition. Dénoncer la vie occidentale par opposition à la référence soviétique est un leitmotiv dans les articles de presse en U.R.S.S. Cette opposition nous permet de mesurer à quel point l'identification du spectateur passe par un appel à la culture individuelle et est de ce fait dangereux pour l'idéologie. La dénonciation du 


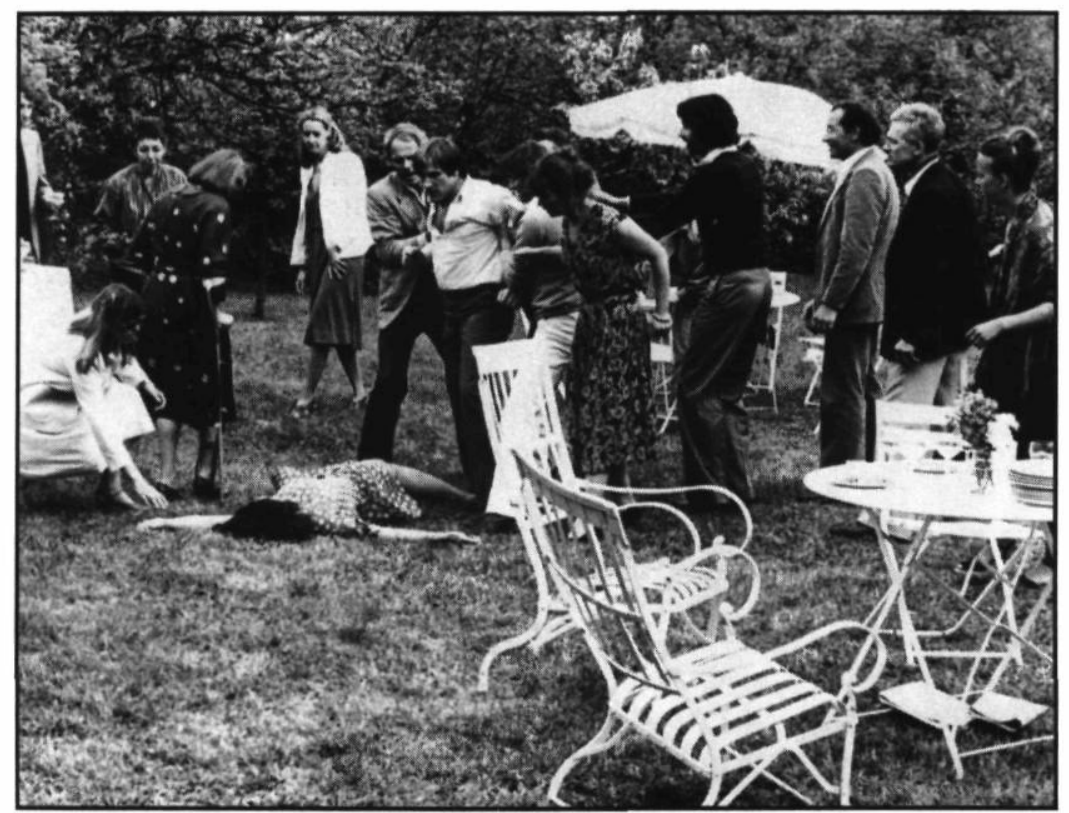

\section{La Femme d'à côfé de François Truffauł (1981)}

Collection Cinémathèque québécoise

régime soviétique, extraite d'un discours de Mihail Romm, cinéaste russe (1901-1971), témoigne de la volonté des autorités de "diaboliser » la culture occidentale '

De façon générale, avant toute analyse des films, il existe un a priori fondé sur une méfiance envers les affirmations occidentales. Les influences extérieures étaient fortement suspectées. C'est un état d'esprit très ancré, qui demeure jusque vers 1987 1988, au moment où les autres pays d'Europe centrale se libèrent, c'est-à-dire au moment où "l'empire soviétique " s'écroule véritablement.

La remise en question des perceptions occidentales, la confrontation systématique avec les points de vue idéologiques soviétiques présente l'idéologie soviétique comme un idéal social. L'apologie de l'idéologie de masse étant un critère d'adhésion insuffisant, il s'agit de montrer que la société capitaliste ne permet pas pour autant l'épanouissement de l'individu. D'où la dénonciation de la bourgeoisie occidentale. Sur le plan cinématographique, la volonté, devenue quasi obsessionnelle à partir 
des années soixante-dix, de concurrencer la machine hollywoodienne allait justement dans ce sens. V. A. Outilov titre ainsi son article: "La crise ${ }^{2}$ de l'idéologie bourgeoise et le cinéma de l'Ouest: la morale de la société de consommation et la désintégration, désagrégation de la personnalité, de l'individu."

On est proche de la citation sur Les Quatre Cents Coups (Truffaut, 1959) parue en 1960 dans un quotidien de Syktyvkar et datant pourtant d'une période de relative libéralisation ${ }^{3}$. Ceci corrobore l'idée que l'individualisme et l'originalité, qui déterminent la stratégie truffaldienne, demeure toujours le danger permanent pour l'idéologie. Par exemple, la conception de l'artiste responsable, du réalisateur-patron, développée dans $L a$ Nuit américaine, accentue l'idée d'individualisme: "Le cinématographe aux U.S.A.: le business par ses moyens préférés" titre Youri Kosmov ${ }^{4}$. Aux yeux de l'idéologie communiste, le cinéma bourgeois (c'est-à-dire non étatisé) pervertit la réalité des masses $^{5}$. Selon elle, le film occidental est exclusivement envisagé en termes de bénéfices. Le but du régime, à travers la plume des critiques, est donc d'annihiler les approches du cinéma "capitaliste ", qui est représenté ici par la stratégie artistique de Truffaut. Ces bases idéologiques sont issues de la révolution d'Octobre 1917 des bolchévistes, comme en témoigne les propos de Léon Moussinac, en décembre 1927-avril $1928^{6}$. Ainsi, la stratégie truffaldienne "contre" totalement l'immobilisme et l'uniformisation qui étaient la garantie de survie de l'idéologie soviétique. Il est donc nécessaire, voire vital, de la dénoncer.

De ce fait, le véritable compliment soviétique est de montrer que le film occidental échappe à une conception capitaliste. Montrer par exemple qu'une actrice, par son attitude et par son jeu, n'adhère pas à la démesure du vedettariat s'inscrit tout à fait dans la conception socialiste de l'art. Dans son article sur Le Dernier Métro, Andreï Plakhov reprend quelques éléments propres à la dénonciation soviétique du star system dans le cinéma occidental. Le titre de l'article est déjà annonciateur: "Catherine Deneuve: Star involontaire."

Lopposition entre la spécificité auteuriste, la prépondérance de l'individu, qui compose la stratégie artistique de Truffaut, et l'idéologie de masse est donc au centre de la menace que con- 
tient toute idée de différence. À partir de là, nous confronterons les analyses critiques du Dernier Métro et de La Femme d'à côté, afin de montrer comment les films peuvent représenter une menace d'autant plus grande que le désir de liberté individuelle est croissant. Ainsi, dans Le Dernier Métro ${ }^{7}$, l'idée de lutte "collective" sera constamment mise en évidence, avant tout pour éviter que le lecteur-spectateur ne s'identifie primordialement à une approche "individualiste" (états d'âme des personnages, etc.).

La référence à la lutte sociale est donc déterminante. Dans cet esprit, l'évocation du fascisme et de l'occupation allemande en France débouche aussitôt sur des considérations politiques. Le régime soviétique s'est d'ailleurs consolidé par sa lutte contre le fascisme (c'est durant la dure période de la guerre que se sont ancrées les bases de la cinématographie soviétique). L'U.R.S.S. mettait souvent en évidence sa lutte et sa victoire sur le fascisme. Le thème de la défense nationale constituait un facteur d'unité largement employé dans les propagandes ${ }^{8}$.

Le fait de privilégier le thème de la Résistance et de l'interpréter avec un regard marxiste a pour vocation d'empêcher le spectateur d'actualiser un sens potentiel et même dominant du film de Truffaut. C'est pourquoi l'interprétation idéologique occulte l'exaltation romantique si chère à la tradition russe. Ce désir de contourner le danger se retrouve à deux niveaux.

Premièrement, la véracité de la reconstitution historique constitue un critère primordial d'appréciation. C'est par cet élément que commence le chapitre de Galina Dolmatovska :

François Truffaut et sa co-scénariste Suzanne Schiffman ont étudié pendant plusieurs années les archives témoignant de l'activité culturelle dans le Paris des années d'occupation. [...] Dans les films de Truffaut, rien n'est laissé au hasard, le moindre détail contribue au développement du sujet. Truffaut a glissé beaucoup d'éléments véridiques dans son film. Par exemple, l'épisode où l'acteur principal engagé par Marion Steiner (joué par Gérard Depardieu) règle son compte au critique fasciste qui avait attaqué le spectacle fait directement référence à une histoire équivalente survenue entre Jean Marais et le critique collaborationniste, Alain

La réception du Dernier Métro et de La Femme d'à côté en U.R.S.S. 209 
Laubreaux, qui s'en était pris à Jean Cocteau. Le Paris nocturne aux vitres bleutées des cafés et des réverbères est bien lui aussi celui de ces années-là (p. 266).

L'interprétation se situe bien dans la volonté soviétique de modifier et de justifier toutes les évocations, ce qui reprend l'opposition entre réalisme et romantisme, qui s'était atténuée au début des années soixante?

La tentative de la part de la critique soviétique de réduire la distance entre l'horizon d'attente idéologique et l'œuvre de Truffaut s'appuie aussi sur un second système de références. L'insistance sur le thème de l'antisémitisme dans Le Dernier Métro conforte le fondement même de la société soviétique, creuset multiracial uni autour d'une même idéologie. Le titre de l'article du journaliste russe Félix Andreiev: "Le salut dans la lutte. Examen des années antifascistes / XII Festival de Moscou " témoigne du rôle du fédéralisme dans la lutte marxiste. Lappel au renforcement de l'unité nationale est net dans les années soixante-dix et quatre-vingt, les discours des dirigeants l'attestent $^{10}$. Le fédéralisme était vigoureusement loué, en particulier durant l'ère Brejnev. La doctrine marxiste qui s'appuie sur l'intégration des particularismes culturels détermine l'orientation de la critique. En ce sens, l'ambition profonde de cette orientation est la réduction du caractère proprement artistique de l'œuvre. L'activité réceptrice n'est plus obligée de se réorienter vers une expérience étrangère à son cadre de vie. L'idéologisation contrarie toute tentative de "changement d'horizon", mais comble par contre l'attente suscitée par les normes idéologiques (noter la première phrase de l'article suivant où le critique appelle le spectateur à envisager Le Dernier Métro différemment des autres films de Truffaut) :

Le Dernier Métro est avant tout important en ce que Truffaut, qui affirmait si souvent dans les interviews son indépendance à l'égard de la politique, est cette fois intervenu dans le débat sur l'occupation et la Résistance. En faisant de l'un des personnages principaux du film un héros de la Résistance et en accordant nettement ses sympathies à Bernard Granger, Truffaut a de ce fait déterminé sa position sur le rôle actif de la Résis- 
tance française, qui s'est aussi manifestée dans le domaine de l'art. De plus, toute la manière de travailler du thêâtre Montmartre incarne la défense des hautes traditions artistiques, la préservation de la culture nationale, la Résistance spirituelle qui fait honneur aux Français. Truffaut, par l'intermédiaire de Lucas, exprime (ce qu'il a d'ailleurs confirmé dans la presse) son respect pour les artistes français qui, dans un pays assiégé en proie à des persécutions racistes, ont continué à créer - comme, par exemple, Alexandre Trauner ou Joseph Kosma (les compagnons de Marcel Carné, avec lequel ils ont réalisé Les Visiteurs $d u$ soir et Les Enfants du paradis).

Ce sont des faits de société français qui ont, comme cela arrive souvent, favorisé le succès du Dernier Métro et son interprétation d'un point de vue politique. En effet, par un malheureux concours de circonstances, la première du film a eu lieu le 3 octobre 1980, le jour même où une bombe explosait devant la vieille synagogue de la rue Copernic. Le film, qui dénonce l'antisémitisme dans la France de Vichy et de l'occupation, s'est trouvé en phase avec l'indignation des Français face aux agressions racistes des années 1980. Ceci a contribué à en faire un événement non seulement de la vie culturelle, mais également de la vie sociale du pays". Truffaut a poursuivi la tradition des films de guerre qui affirmaient la détermination et la grandeur de l'esprit français. Il l'a poursuivie avec des larmes et des sourires, comme le veut une comédie dramatique, dans laquelle la vie de thêâtre et la réalité, curieusement, sont appelées à se rejoindre. Le Dernier Métro est un film "intérieur ", fait pour les Français et c'est pour cette raison qu'il les a tant interpellés (Dolmatovska, p. 269-270).

Le thème de l'identité nationale uniquement envisagée autour d'une même idéologie est ici mis en évidence. Aux yeux du critique soviétique, Truffaut s'engage et loue les valeurs de la Résistance. Par là même, le film revêt un caractère documentaire en trouvant son authenticité autour de l'histoire de la France et acquiert sa cohésion dans un élément précis de la réalité sociale spécifiquement nationale et idéologique.

L'interprétation sociale, la portée historique et idéologique est donc privilégiée par rapport aux sentiments. L'attention pour le 
"facteur humain" n'est pas conciliable avec la conception soviétique qui affirme que l'homme est un élément dans le moulin social et étatique. Cette conception était très ancrée dans les mentalités et le fut encore pendant très longtemps, comme le note $S$. Kitchiguine :

Peu se sont libérés de l'idéologie héritée de Staline : l'individu n'est qu'une vis [...] et nous trouvons parfaitement naturel que cette unité, l'être humain, soit au service du groupe, de l'entreprise, que chacun exploite l'autre [...] Ce qui prédomine, c'est une attitude indifférente à l'égard "du facteur humain».

Par exemple, pour "justifier" la raison pour laquelle Marion tombe amoureuse de Bernard, la journaliste soviétique invoque la Résistance à laquelle appartient Bernard comme unique motivation:

Peu à peu, Lucas comprend que Marion s'est éprise de Bernard, non seulement parce qu'il a osé tenir tête au critique nazi, mais parce qu'il est un véritable héros de la Résistance: il fabrique lui-même des bombes pour les clandestins. Bernard quittera assez vite le théâtre pour le maquis, et n'y reviendra qu'après la Libération (Dolmatovska, p. $268^{12}$ ).

La tentative de la part du critique de combler l'attente suscitée par les normes idéologiques se poursuit ici. En effet, les relations entre Marion et Bernard sont décrites avec beaucoup plus de subtilité et les instants de vérité n'en sont que plus percutants. Contrairement à ce qu'affirme le critique soviétique, l'évolution des sentiments de Marion est aussi dissimulée que progressive. Elle est troublée par Bernard bien avant de s'apercevoir qu'il est Résistant. Et, son amour pour lui supplante même sa prétendue admiration pour les activités de Bernard liées à la Résistance. Ainsi, un instant de vérité intervient dans le film: quand Bernard annonce à Marion qu'il quitte momentanément le théâtre pour la Résistance, elle le gifle violemment...

La distinction entre la critique française et soviétique est révélatrice de l'importance du contexte historique. Là où la critique française voit une mise en évidence des péripéties dramatiques et 
amoureuses, la critique soviétique voit un retour à un thème cher de l'enfance de Truffaut, la vie en France sous l'occupation et la Résistance. Le journaliste montre que la presse française fit une grande place aux péripéties dramatiques, à l'amour pour le théâtre et à l'amour tout court, qui constituent la trame du film. Avec des expressions comme "selon nous", "il nous semble", G. Dolmatovska marque bien les deux perceptions différentes, française et soviétique :

Le critique Pierre Billard du journal Le Point voit dans le trio lyrique Lucas-Marion-Bernard la réincarnation des trois personnages de Jules et Jim, non plus au lendemain de la Première Guerre mondiale, mais cette fois-ci durant la Seconde. Nous sommes d'accord avec l'appréciation du critique sur la "fidélité du réalisateur à lui-même" mais, cependant, selon nous, cette fidélité se manifeste autrement. [...] Le Dernier Métro, du point de vue de l'époque concernée, correspond pour Truffaut à un retour à l'adolescence des gens de sa génération. [...] Truffaut a mûrement réfléchi à son film Le Dernier Métro et l'a mis au point avec grand soin. Lui-même en parlait avec beaucoup de retenue: "Le thème principal du film n'est pas la politique. [...] Depuis longtemps, j'avais envie de tourner un film sur le théâtre et j'étais aussi intéressé par l'idée d'un film sur l'occupation. Le Dernier Métro m'a permis d'associer ces deux désirs ». Il n'en reste pas moins que le film a dépassé le cadre d'une histoire de gens de théâtre à Paris en 1942 (p. 269).

Le Dernier Métro occupe toujours une place privilégiée dans les critiques soviétiques parce que le sujet du film contient une réflexion sociopolitique qui permet une interprétation idéologique:

Respect de l'homme, attention soutenue au monde intérieur et complexe du personnage, finesse de l'analyse psychologique, atmosphère poétique, toutes ces qualités-là, nous sommes habitués à les rencontrer dans les films de François Truffaut. En outre, dans ses œuvres les plus remarquables, le réalisateur réussit à déboucher sur les problèmes brûlants de notre temps. Ainsi, dans Le Dernier Métro, présenté au précédent 
Festival de Moscou, était retracé de façon saisissante le Paris de l'occupation; on y trouvait une réflexion sur les possibilités d'une résistance morale au fascisme, au plus profond de la conscience du personnage (p. 150).

Par rapport à cette référence, les autres films de Truffaut vont être sous-estimés, dans la mesure où le "changement d'horizon" peut être plus difficilement enrayé.

L'engagement sociopolitique du metteur en scène est donc présenté comme un motif central d'appréciation des films de Truffaut et notamment dans les critiques du Dernier Métro. C'est la définition de l'artiste marxiste, forcément engagé politiquement au service de l'idéologie: "Le héros principal du Dernier Métro abandonne le théâtre pour lutter dans les rangs de la Résistance et je pense qu'il est légitime d'en déduire que Truffaut, s'il avait été adulte en 1942, aurait agi de même. Sans doute est-ce même précisément cela qu'il a voulu dire pour son film ", affirme A. Braguinski (p. 180).

La coloration marxiste de l'interprétation est d'autant plus marquée qu'elle differe de la façon dont Truffaut présentait ses films. Dans le cas du Dernier Métro, le critique exagère totalement l'apologie de la Résistance, l'engagement idéologique du cinéaste et la notion de responsabilité du citoyen au sein de l'État chez Truffaut, qui demeure avant tout quelqu'un de sensible à "l'individu", à une sensibilité plutôt qu'à une idée ou un groupe. C'est un trait de caractère qui se retrouve dans toute sa conception morale et cinématographique; d'où une obligatoire reconstruction idéologique de son œuvre dans la réception critique soviétique ${ }^{13}$.

La théorie marxiste "l'existence détermine la conscience" oriente les critiques. Le but profond est de réduire au maximum toute complexité, toute ambiguïté, forcément plus présente dans une conscience individuelle que dans une idéologie collective. On ne peut, en ce sens, adhérer à plusieurs points de vue individuels.

La stratégie de Truffaut, en privilégiant l'individu, privilégie l'ambivalence et la complexité psychique; elle représente donc une menace pour l'idéologie marxiste. D'où les analyses critiques de La Femme d'à côté ${ }^{14}$ qui, faute de faire l'objet d'une 
reconstruction possible (étant donné l'absence de connotation socio-politique), sera totalement dénoncé, ce qui ne manquera pas de renforcer le goût de l'interdit chez le spectateur soviétique.

Contrairement au Dernier Métro, qui ne fit pas l'objet d'une distribution commerciale, La Femme d'à côté fut le troisième film distribué en U.R.S.S. Cependant, alors que Le Dernier Métro fut généralement bien perçu par la critique, La Femme d'à côté connut un grand succès public mais un échec critique. La distorsion qui existe entre la réception critique du Dernier Métro et de La Femme d'à côté d'une part et entre la réception publique et critique de La Femme d'à côté d'autre part confirme la volonté soviétique d'annihiler tout danger inhérent à l'individualisme :

François Truffaut aime, dans ses films, prendre comme point de départ des situations à dessein banales, pour leur conférer ensuite un tour inattendu. C'était le cas pour Jules et Jim, La Peau douce et quelques autres, où le réalisateur réussissait à accomplir son projet avec une indispensable force artistique de persuasion. Dans $\mathrm{La}$ Femme d'à côté, le banal tragique semble intentionnel, prémédité, il est faiblement motivé; les motifs ne sont pas clairement exposés. Voici 15-20 ans, l'art de l'Occident tirait le signal d'alarme à propos du "manque de spiritualité " de la vie bourgeoise. Le point de vue est aujourd'hui différent, et Truffaut, tout pareillement, regarde d'un autre œil ce phénomène social : il observe le vide social de ses personnages simplement comme une donnée, comme une "anormalité", dans son genre "normal". C'est pourquoi le drame de l'amour qu'il a représenté a perdu ses proportions, son échelle : ce n'est pas la protestation d'une personnalité vigoureuse, ni l'expression d'une soif spirituelle, c'est simplement le fruit de sentiments exacerbés et de nerfs détraqués (Bojovitch, p. 151-152).

L'art soviétique doit décrire la vie sociale en insistant bien sur le fait que cela ne se limite pas à des descriptions psychologiques sentimentales ou à des individus. Dans la conception soviétique, tout est lié à l'élaboration du socialisme et de ce fait, la lutte contre les "vices capitalistes" est permanente. C'est pourquoi, Victor Bojovitch écrit : 
Dans La Femme d'à côté, le champ de vision est à dessein rétréci. Un quartier résidentiel proche de Grenoble [dans le texte: banlieue bourgeoise], deux familles de cadres supérieurs [mot à mot: d'employés qui ont réussi], la passion qui éclate de façon brutale, et aucun problème, sauf la complexité et les nuances des rapports amoureux ( p. 150).

Chaque film doit présenter une question, une idée, révéler un problème réel ou actuel. Les films sont évalués en fonction de leur pouvoir social et révolutionnaire, ce qui contraint le critique soviétique à se demander :

Qui sont ces gens entraînés dans un drame d'amour dont les péripéties sont recréées de façon la plus minutieuse, dans La Femme d'à côté? Avant toute chose, ils n'ont aucun problème d'ordre matériel et moral. Ils n'ont pas d'intérêt pour leur profession: leur travail, ce n'est rien de plus qu'un moyen de gagner de l'argent, d'assurer à leur famille un niveau de vie indispensable (Bojovitch, p. 151).

Jusqu'à la perestroïka, des termes comme "niveau de vie " étaient réservés aux analyses des conditions de vie des travailleurs des pays capitalistes, d'où la constante dénonciation du mode de vie occidental. Le facteur humain est un rouage au service des tâches collectives déterminées par le Parti. Les milieux de travail suscitent beaucoup d'intérêt dans les descriptions socialistes: le travail est un grand catalyseur des conflits personnels.

En conséquence, la psychologie fut toujours très attaquée et on prône, après la perestroïka, un retour à celle-ci. Dans une interview au journal Troud ${ }^{15}$, B. F. Lomov, directeur de l'Institut de Psychologie, dresse la situation morose de sa discipline en U.R.S.S. : "Le Système administratif n'avait nul besoin de la psychologie en tant que science des lois de l'activité psychique, de son développement et de ses diverses formes. Là où prévaut un style autoritaire, il n'y a pas de place pour l'étude, l'analyse et la prise en compte de l'individualité. " Le problème de l'homme soviétique, privé de liberté, c'est qu'il ne peut pas être maître de son destin. Ici encore, l'idéologie soviétique prend ses distances 
avec la tradition humaniste chrétienne (la médecine russe des deux emstvos: tact, pitié et sagesse) qui s'oppose à l'humanisme marxiste :

Les liens entre les personnages sont déterminés par le hasard; partage d'idées ou divergences, penchants ne jouent aucun rôle. Les événements de leur entourage, de la planète, n'atteignent en aucune façon ni ne troublent les héros. Ils mènent une vie légère, monotone et quelque peu inconsistante. Dans ces conditions, l'inclination amoureuse qui naît, qui éclate entre un homme et une femme, les prend tout entiers; le plaisir et la satisfaction procurés par cette attirance constituent l'unique but de leur vie. Les héros du film sont des gens normaux, mais ils se conduisent comme des obsédés. Mathilde aboutit dans un service de neurologie; à sa sortie de clinique, elle tue son amant et se suicide, agissant sous l'emprise de la pathologie (Bojovitch, p. 151).

La stratégie artistique de Truffaut, qui donne toute-puissance à l'individu évoluant souvent en dehors des normes afin de donner libre cours à ses passions personnelles, s'oppose à l'idéologie marxiste et est, en ce sens, combattue. Les répressions qui apparurent notamment après 1968 avaient pour but d'éviter les débats, polémiques, recherches et innovations. Pour les autorités soviétiques, la société est homogène et ne doit pas être traversée par des antagonismes entre classes, couches sociales ou individus. Les autorités se méfiaient du film, susceptible d'être un moyen de réflexion publique. Comme l'écrivain Stephan Hermlin le déclara (dans une interview en français de novembre 1982) : "Les marxistes ont toujours aimé les classiques, les gens pour ainsi dire sans crise, bien réglés" (cité dans Laveau, 1985, p. 209). Le critique soviétique dénonce donc le caractère névrotique des personnages de La Femme d'à côté.

Dans la tradition russe, l'approche psychiatrique était caractérisée par une grande tolérance envers les problèmes psychiques. La volonté réelle d'aider l'autre était au centre de la tradition humaniste. Cette tradition est reniée dans les années trente par le régime ${ }^{16}$. La psychiatrie était un sujet tabou, les problèmes mentaux étaient impossibles dans les régimes socialistes. D’où la 
non-officialisation de la psychiatrie, comme méthode médicale. Sa seule évocation consistait à l'utiliser pour dénoncer le mode de vie occidental.

Toujours vécues comme un élément perturbateur, toutes les scènes à connotation sexuelle étaient dénoncées. La sensualité n'était pas considérée comme une attitude saine dans l'idéologie marxiste. Dans les motifs de censure, la sexualité tient une place primordiale, toujours parce qu'elle est susceptible de diminuer les thèmes révolutionnaires et collectifs ${ }^{17}$. En témoigne la citation précédente qui fait écho à celle du passage suivant: «[...] des individus privés de points de référence personnels, d'axes, de pivots, qui cèdent à leurs pulsions inconscientes trop facilement ${ }^{18}$ ". La sexualité était toujours réprimée, vécue comme un vice proprement bourgeois, alors que la société soviétique trouve ses sensations fortes dans la lutte idéologique. Le thème de la sexualité étant largement présent dans l'œuvre de Truffaut, et en particulier dans La Femme d'à côté, il existe aussi à ce niveau un motif de menace idéologique.

Toutes les critiques de La Femme d'à côté sont donc régies par trois critères de dépréciation: elles s'opposent à la complexité psychologique, au pessimisme et au manque de motivation sociale et idéologique des personnages. C'est tout à fait dans l'esprit des dirigeants soviétiques, comme en témoignent les propos des censeurs du Goskino réprouvant des aspects particuliers du film Le Miroir (1974), d'Andreï Tarkovski, l'un des auteurs les plus originaux et spirituels du cinéma soviétique ${ }^{19}$.

Ainsi, l'exigence de clarté et de simplification à la fois esthétique et morale, prônée par le Goskino, fait écho aux interprétations de La Femme d'à côté dénonçant la "complexité et nuances des rapports amoureux". Deuxièmement, le pessimisme est dénoncé comme dans les critiques sur La Femme d'à côté. Troisièmement, la nécessité de motivations et de messages socioidéologiques prônée par le Goskino se retrouve dans les termes critiques sur le film de Truffaut, tels que "vide social de ses personnages", "champ de vision à dessein rétréci", "Qui sont ces gens?", "ce drame de l'amour a perdu ses proportions, son échelle", "le finale est faiblement motivé, les motifs ne sont pas clairement exposés", "liens déterminés par le hasard ", "Ils n’ont 
pas d'intérêt pour leur profession", "obsédés, pathologie", "inconsistance d'amants tragiques". Il ne faut pas montrer que les personnages ont "vécu leur vie en vain" ou que des séquences " ne sont motivées par rien".

Les deux extraits suivants confirment l'évolution idéologique de l'U.R.S.S. après la perestroïka, la réhabilitation du domaine privé, de l'amour, de la passion, du sexe:

L'Homme qui aimait les femmes, c'est le titre de l'un des meilleurs films du grand réalisateur français François Truffaut. Et on pourrait le dire de Truffaut lui-même, lui que les critiques ont appelé "le plus pudique et le plus érotique de tous les réalisateurs français [...] ".

Il serait aisé de se convaincre de la justesse de ces mots ne serait-ce que par les quelques films qui sont passés sur nos écrans: il y a une vingtaine d'années, L'Argent de poche et Les Quatre Cents Coups, et récemment, $L a$ Femme d'à côté et Vivement dimanche! Nos spectateurs se sont attachés à Truffaut même à travers cette parcelle de son œuvre. Et, ils ont assailli le ciné-vidéo-club d'Odessa de lettres demandant la rediffusion de $L a$ Femme d'à côté, souhait qui n'a pu, hélas, être exaucé, la copie existante n'étant plus en état d'être projetée (Vladimirskaja).

Le choix de deux films différents comme Le Dernier Métro et La Femme d'à côté, l'un comportant un fond historique et politique important, l'autre étant complètement passionnel, fut révélateur de la constance idéologique que la menace individualiste contenue dans le style de Truffaut oblige à développer. L'“effet de l'œuvre" doit être orienté ou dénoncé dans le but d'empêcher le "changement d'horizon", c'est-à-dire que des expériences esthétiques nouvelles accèdent à la conscience réceptrice.

L'étude soviétique révèle aussi la distorsion qui exista entre les attitudes du public et celles exigées par les dirigeants. Le totalitarisme culturel créa une véritable fracture entre la culture réelle et l'idéologie officielle; entre les attentes du public et les directives politiques. Le cinéma était de ce fait un lieu de fonctions doubles, officielles et officieuses (propagande politique mais aussi ouverture sur l'Occident). 
La critique soviétique avait donc pour but d'empêcher le spectateur d'actualiser un sens potentiel, voire dominant des films de Truffaut. En même temps, le discrédit jeté sur $\mathrm{La}$ Femme d'à côté ne fut pas réellement efficace puisque les réactions du public soviétique furent très favorables au film de Truffaut. Les films de Truffaut se prêtent particulièrement bien aux appropriations multiples et ambivalentes. La fracture de la société soviétique constitue un terrain très réceptif à l'" effet de l'œuvre" de Truffaut. Les films de Truffaut témoignent d'une même ambivalence, qui leur permet de contenir deux approches parallèles (et non consécutives): une apparence "civile" et une face cachée, personnelle et illicite.

\section{Université d'Aix-Marseille I}

\section{NOTES}

1 «Pourquoi, comme dans le passé, nous attachons-nous encore tant à la soi-disant priorité dans tous les domaines? [...] En défendant, et parfois en inventant, cette primauté à tout prix, qui sait où nous aurions été finir il y a 10 ans, quand nous nous efforcions de nous séparer complètement de la culture occidentale au nom de la "tradition". Nous avons perdu l'habitude de penser qu'en Occident, il existait quelque chose. Et au contraire, jadis, la Russie était le pays du monde où l'on traduisait plus de littérature étrangère que dans n'importe quelle autre nation. L'un des points forts de l'intelligentsia russe résidait justement dans le fait qu'elle lisait toute la littérature mondiale. Cela aussi, c'est une tradition de chez nous. Une excellente tradition qu'il vaut aujourd'hui la peine de reprendre, de se rappeler. Des années durant, on a considéré le néoréalisme italien comme quelque chose dont il fallait se méfier, rien que parce qu'il venait de l'Occident. [...] Nous, alors, nous traversions la phase la plus grave du stalinisme, nous condamnions le "cosmopolitisme sans patrie" et nous cherchions à éloigner de nous l'expérience du néoréalisme italien, en endommageant notre travail.

Rappelons-nous au moins cette vérité, pour pouvoir comprendre en quel monde nous vivons! Et aujourd'hui, quand le groupe qui jadis condamnait à la "mort civile" les "cosmopolites sans patrie", Kocetov, Sofrenov et d'autres, accomplissent une diversion évidente, attaquent tout ce qui se fait de bon et d'avancé dans le cinéma soviétique, il me semble que maintenir, en ce moment, un calme académique et attendre ce qui arrivera, soit une chose à ne pas faire. (Applaudissements prolongés). L'attaque a commencé sur le cinéma, mais je crains qu'elle ne touche d'autres secteurs si l'on ne fait pas taire ces gens" (cité dans Jean-Loup Passek, Le Cinéma russe et soviétique, Paris: Centre Georges Pompidou/L'Équerre, 1981, p. 88-89).

2 La vision idéologique d'un monde bipolarisé entre le capitalisme et le socialisme, était notable dans le vocabulaire, dont certains mots étaient réservés pour chaque système politique, l'un bon, l'autre mauvais. Le terme de "crise" figure parmi ces mots. Depuis les années trente, l'idéologie officielle a toujours loué le fait que l'U.R.S.S. ne connut jamais de crises, ni sociales ni économiques. La planification permettait un progrès continu. Les crises sociales qui ont eu lieu dans les autres pays d'Europe cen- 
trale étaient présentées comme des phénomènes isolés et surmontés grâce à "l'aide fraternelle de l'U.R.S.S.". Le mot "crise" était donc tabou. Il n'était employé que pour qualifier la situation occidentale. Avec la perestroïka, ce mot, comme beaucoup d'autres mots, sera réintroduit.

3 “La voilà, la vie dans la France d'aujourd'hui, disent les auteurs du film. Que vous le vouliez ou non, notre pays est le théâtre de la décomposition de la personnalité humaine. Le système d'éducation lui-même ne voit pas l'homme en tant qu'homme", affirme L. Bortvina dans un "Un cri que personne n'entendra" (Krasnoe Znamja, 23 novembre 1960).

4 L'article évoque en particulier La Nuit américaine et le fait qu'il ait " [...] été considéré comme le meilleur film de l'année 1973, au cours de l'enquête annuelle des membres du cercle des critiques de cinéma new-yorkais" (p. 170). Le journaliste fait en fait allusion à l'oscar du meilleur film étranger à Hollywood.

5 Ce qui est mis en évidence, c'est toujours la dénonciation d'une classe bourgeoise uniquement soucieuse de ses propres intérêts individuels. On cherchait à décompartimenter la création artistique, par le biais de la théorie de masses. La culture soviétique tentait de casser les données sociales et groupementales traditionnelles. Le système d'exploitation cinématographique était très différent de celui des autres pays occidentaux. Le Sovkino détenait le monopole de l'exploitation des films russes ou étrangers sur tout le territoire de l'U.R.S.S. Il était directement administré par le Comité central du Parti communiste.

6 "La rationalisation du travail autorise une production qui dépassera, l'an prochain, celle des pays européens plus ou moins colonisés par l'Amérique, au point de vue cinématographique. Et voilà où le cinéma soviétique apparaît avec tout son caractère et sa prodigieuse activité: logiquement et, bientôt, dans très peu de temps, à condition que ce soit un temps de paix extérieure (quelques années ne comptent guère dans la naissance d'un monde), le cinéma soviétique se trouvera seul en face du cinéma capitaliste international, c'est-à-dire que lui, uniquement, rival sur le plan économique de l'industrie cinématographique des États-Unis, sera capable de lutter contre l'intoxication de la bêtise américaine et l'action de la propagande européenne, en opposant de jeunes œuvres, saines, fortes, rayonnantes, aux films à "combines" des "mercantis" ", écrit Léon Moussinac en 1927-1928 (Le Cinéma soviétique, Paris: Éditions d'aujourd'hui, 1976, p. 174-175).

7 Nous nous appuierons notamment sur l'extrait d'un livre: celui de Galina Dolmatovska, "Le cinéma français: compte-rendu des années de guerre, François Truffaut ", Remarques sur le passé (Moscou: Iskousstvo Kino, 1983, p. 266-270).

8 De nombreux articles ou livres évoquent le cinéma français, essentiellement à travers l'étude des films sur la Seconde Guerre mondiale et donc sur la dénonciation du nazisme, comme le fait l'extrait de l'auteur russe Galina Dolmatovska : «Le cinéma français: compte-rendu sur les années de guerre", Remarques sur le passé (Moscou: Iskousstvo Kino, 1983, p. 266-270). Le public russe apprécie beaucoup les films historiques. Néanmoins, la doctrine politique pousse à l'éducation des masses en privilégiant la Révolution et en dénonçant tout ce qui lui a porté atteinte, en particulier le fascisme, ou de façon plus idéologique, la conception capitaliste.

9 Lanalyse devenait même symboliste, comme la réception des Quatre Cents Coups en U.R.S.S. en témoigne.

10 La Grande Encyclopédie soviétique, en page 255, donne à "fédéralisme" le but suivant: "résoudre la question nationale». En remplaçant l'appartenance nationale et religieuse par l'appartenance idéologique, le régime soviétique abolit toute forme de racisme fondée sur la première appartenance (cité dans Michel Niqueaux (direction), Vocabulaire de la perestroika, Paris / Caen : Éditions universitaires-Centre de recherche sur l'évolution de l'U.R.S.S. de l'Université de Caen, 1990, p. 97). 
11 Il est intéressant de noter le lien que fait le critique avec l'attentat antisémite afin de renforcer le poids sociopolitique du film, dans l'esprit soviétique. C'est d'autant plus délibéré que le rapprochement est un peu forcé puisque la sortie commerciale du Dernier Métro s'effectua en fait le 17 septembre 1980. Il est certes vrai que cet attentat a peut-être développé l'attention des Français pour Le Dernier Métro. Il est intéressant de noter de toute façon que la comparaison n'a pas été notée, à ma connaissance, dans les articles français et étrangers. Il n'est pas négligeable que ce soit justement le fait d'un article soviétique.

12 C'est tout à fait dans l'esprit de l'interprétation soviétique de La Femme d'à côté. La principale raison utilisée pour dénoncer ce film est le fait que la relation amoureuse entre Mathilde et Bernard n'est pas "explicable", "justifiable", elle est purement passionnelle.

13 "Je n'ai jamais vu à Fahrenheit 451 un sens politique, je n’ai jamais pensé que c'était la condamnation de la dictature. Il y a une chose que j'ai beaucoup aimée dedans et qui correspond probablement à mes idées sur la vie: c'est la notion de résistance rusée. On vous interdit d'avoir des livres et même on les brûle, alors, pour sauver la culture, des types apprennent des livres par cœur; cela consiste à contourner la loi, à ne pas s'y opposer ouvertement, l'important étant de gagner, c'est une idée précieuse pour moi. [...] Alors il y avait cela dans Fahrenheit 451, cette dissimulation qui m’a touché et qui était tellement... dissimulée que personne ne l'a vue! Le héros lit des livres en cachette, subrepticement, comme je voyais, enfant, les films en cachette. J'ai toujours tout fait en cachette dans ma vie. [...] Ce ne sont pas des résistants. Je n'aime pas la Résistance. Je ne sais pourquoi. J'ai eu des résistants dans ma famille. Enfin, j'ai eu une famille où se trouvaient à la fois des collaborateurs et des résistants, mais je n'admirais ni les uns ni les autres. Lidée de la Résistance ne me plaisait pas, l'idée qu'une partie de la France était “dans le vrai". Je n'aime pas que l'on fasse partie de quoi que ce soit, en fait. Je me souviens qu'à 13 ans, en lisant les comptes rendus du procès Pétain, je pensais à ce vieux, tout seul, qui avait soudainement tous les torts, abandonné par tout le monde... et tous les autres, là, qui représentaient la justice. Je n’aimais pas ça. [...] Je trouve impossible d'introduire des réactions émotionnelles dans un problème politique. Il n'y a pas les brebis et les loups. Pour moi, ce sont des loups des deux côtés. [...] Je n'aime pas l'idée d'un bloc solide, uni parce que ses habitants savent qu'ils ont raison. [...] Il m'est impossible d'éprouver de l'amitié pour un peuple en bloc, impossible. L'amitié, ça marche avec les personnes, une par une, pas avec les groupes. Je me sens étranger à toutes les idéologies. J'essaie de ne pas avoir affaire aux "officiels". Un vrai cinéaste de gauche doit souhaiter que le cinéma dépende de l'État, que la culture soit subventionnée. Je préfere que les films se financent tout seuls ou du moins se rentabilisent sans le secours de l'État. Je préfere le jeu normal du commerce, fabriquer un film et le vendre avec un bénéfice. Autrement dit, si le cinéma français devait se nationaliser un jour sous quelque régime que ce soit, socialiste ou "libéral", comme celui que nous avons actuellement, je crois que ce serait pour moi un motif suffisant pour partir m'installer ailleurs, dans un pays où le cinéma dépend du système capitaliste des dépenses et des profits", affirme François Truffaut dans " 46 réponses de François Truffaut à 47 questions de Pierre Ajame ", Le Nouvel Adam, n 19 (1968), p. 30-31.

14 Contrairement au Dernier Métro, le choix de La Femme d'à côté est particulièrement intéressant, car de par son sujet, il s'inscrit tout à fait dans la tradition des romans passionnels du XIX ${ }^{\mathrm{e}}$ siècle, très appréciés en Russie. Le film nous permet donc aussi de mettre en évidence l'opposition entre la culture et l'idéologie.

15 Quotidien d'information générale, gouvernemental, ayant à cette époque le plus fort tirage de la Russie: 15700000 exemplaires.

16 C'est en effet à partir de la campagne de désinformation consécutive au congrès d'Honolulu (septembre 1977) de l'Association mondiale de psychiatrie (où les abus 
de l'U.R.S.S. furent dénoncés) que la répression contre la psychiatrie fut renforcée. Tout ce qui a trait au psychisme était gardé sous silence en U.R.S.S. : "Chez nous, il n'était pas d'usage d'aborder le thème de la psychiatrie: les médecins aussi bien que la presse respectaient la "loi du silence" ", affirme le ministre de la Santé Potapov en 1988 (Sovefskaia Rossia, 30 mars 1988).

17 Toutes les activités privées, y compris la vie sexuelle, devaient être négligées par rapport à l'élément principal: I'activité révolutionnaire et collective. Le "cocon" familial était en effet considéré comme une vie bourgeoise, typiquement capitaliste. L'État contrôlait le domaine privé. Les Soviétiques sont, comme le souligne Alexandre Dimov en 1980, des "hommes doubles". Le sexe et le psychisme sont refoulés et on cherche des compensations de façon furtive et clandestine; c'est bien connu depuis Freud: "Dans la deuxième édition de la Grande Encyclopédie soviétique (1976), l'article "Vie sexuelle", rédigé par le sociologue "libéral" Igor Kohn, contient encore la référence obligée à Lénine et rappelle au lecteur soviétique que "malgré toute leur intimité, les relations entre les sexes sont avant tout sociales", ce qui résume sans ambiguïté le point de vue officiel. Par contre, on y trouve enfin le mot "sexe", interdit par la censure jusque vers la deuxième moitié des années soixante (mais ainsi décrit) : "mot d'origine française, employé dans la langue russe contemporaine dans le même sens que sexualité" "(Michel Niqueux (direction), Vocabulaire de la pereströ̈ka, Paris / Caen : Éditions universitaires-Centre de recherche sur l'évolution de l'U.R.S.S. de l'Université de Caen, 1990, p. 198).

18 "L'inconsistance de caractère, la faiblesse apparaît, peut-on dire, comme le signe distinctif et caractéristique des personnages évoqués par Truffaut. Le metteur en scène tire ses héros sans volonté et sans éclat, sans force de caractère, vers le rôle d'amants tragiques. Mais indépendamment de son intention, il en résulte un film sur une vie dénuée de sens, insignifiante, sur des individus privés de points de référence personnels, d'axes, de pivots, qui cèdent à leurs pulsions inconscientes trop facilement pour qu'à leur suite, on puisse discerner la vérité sur la tragédie de l'amour ", souligne Victor Bojovitch dans "La Voisine", Iskousstvo Kino, n" 12 (1983), p. 151-152.

19 "Le Goskino de l'U. R. S. S. juge indispensable de faire les remarques suivantes à propos du Miroir de Tarkovski :

"La complexité de la construction visuelle, la superposition des liaisons entre les différents épisodes exigent avant tout que l'auteur clarifie la logique de sa pensée et l'action du film dans son ensemble. De notre point de vue, il faut accorder une attention particulière à recomposer et corriger toute une série de scènes et d'épisodes. [...] Il serait souhaitable de retravailler l'épisode "Espagne" afin de gommer la tonalité trop triste de l'épisode; il faut davantage souligner la joie éprouvée par les enfants espagnols lorsqu'ils arrivent en U.R.S.S. Les séquences documentaires avec les aérostates ne sont motivées par rien, elles sont donc inutiles. [...] Le texte, lu par le narrateur à la première personne, est trop pessimiste. Il donne l'impression que l'artiste, au nom duquel se fait le récit, a vécu sa vie en vain, qu’il n’a pas réussi à faire passer son message à travers l'art. Pour effacer cette impression, il est nécessaire d'introduire dans le texte quelques répliques nouvelles" (Fomine, Valeri et al., cité dans U.R.S.S., 50 ans de cinéma retrouvé, Paris: La Cinémathèque française, 1990, p. 14-15).

\section{OUVRAGES CITÉS}

Andreiev, Félix. "Le salut dans la lutte. Examen des années antifascistes / XII" festival de Moscou ". Culture soviétique (21 juillet 1981) p. 5-15.

Bojovitch, Victor. "La Voisine». Iskousstvo Kino, n" 12 (1983) p. 149-152.

Bortvina, L. "Un cri que personne n'entendra ». Krasnoe Znamja (23 décembre 1960).

La réception du Dernier Métro et de La Femme d'à côté en U.R.S.S. 
Braguinski, Alexandre. "Souvenirs de François Truffaut, réalisateur français 19321984 ". Iskousstvo Kino, n' 2 (1985) p. 179-180.

Dimov, Alexandre. Les Hommes doubles, la vie quotidienne en Union soviétique. Paris: Éditions J. C. Lattès, 1980.

Dolmatovska, Galina. "Le cinéma français: compte-rendu des années de guerre, François Truffaut ", Remarques sur le passé. Moscou: Iskousstvo Kino (1983) p. 266270.

Fomine, Valeri, Plakhov, Andrë, Roubanova, Irina, Yampolski, Mikhaïl. U.R.S.S., 50 ans de cinéma retrouvé. Paris: La Cinémathèque française, 1990.

Kitchiguine, S. L'Envol de la pomme. Kiev: Éditions Molod, 1989.

Kosmov, Youri. "Le cinématographe aux U.S.A. : le business par ses moyens préférés ". Iskousstvo Kino, n 1 (1975) p. 169-180.

Laveau, Paul. La RDA au quotidien. Paris: Messidor-Éditions sociales, 1985.

Lomov, B. F. «Interview ». Troud (3 janvier 1989).

Moussinac, Léon. Le Cinéma soviétique. Paris: Éditions d'aujourd'hui, 1976.

Niqueux, Michel (direction). Vocabulaire de la perestroïka. Paris / Caen : Éditions universitaires-Centre de recherche sur l'évolution de l'U.R.S.S. de l'Université de Caen, 1990.

Outilov, V. A. "La crise de l'idéologie bourgeoise et le cinéma de l'Ouest: la morale de la société de consommation et la désintégration, désagrégation de la personnalité, de l'individu ". VGIK(1982).

Passek, Jean-Loup (direction). Le Cinéma russe et soviétique. Paris: Centre Georges Pompidou/L'Équerre, 1981.

Plakhov, Andreï. "Catherine Deneuve: star involontaire». Ciné-Riga, n" 11 (1982), p. 27-29.

Potapov. SovetskaiaRossia (30 mars 1988).

Truffaut, François et Ajame, Pierre. " 46 réponses de François Truffaut à 47 questions de Pierre Ajame ". Le Nouvel Adam, n 19 (1968) p. 24-32.

Vladimirskaja, G. "En prévision de la rétrospective, L'Homme qui aimait les femmes ». Odesskij Vestnik (1" mars 1994). 